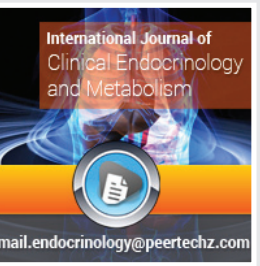

Clinical Group

\title{
International Journal of Clinical Endocrinology and Metabolism
}

\section{Ashutosh Kakade, Ipseeta Ray Mohanty* and Sandeep Rai}

Department of Pharmacology, Department of Medicine, MGM Medical College, Navi Mumbai, India

Dates: Received: 16 February, 2017; Accepted: 16 March, 2017; Published: 17 March, 2017

*Corresponding author: Ipseeta Ray Mohanty, Professor, Department of Pharmacology, MGM Medical College, Kamothe, Navi Mumbai, Maharasthra, 410209, India, Tel: 91-981 9908498; Fax: 91-2256661988; E-mail: ipseetamohanty@yahoo.co.in

Keywords: Type II diabetes mellitus; Antidiabetic prescription; Drug utilization

https://www.peertechz.com
Research Article

\section{Assessment of Prescription Pattern of Antidiabetic Drugs in the Outpatient Department of a Tertiary Care Hospital}

\section{Abstract}

Background: Glycemic control remains the major therapeutic objective for prevention of target organ damage and other complications arising from diabetes. Poor glycemic control in diabetes mellitus can be prevented by using rational use of oral hypoglycemic agents $(\mathrm{OHA})$. Rational use of the drugs in populations can be effectively evaluated using drug utilization studies.

Method: Necessary approval from the Institutional Ethics Committee was obtained before initiating the study. The study was conducted in the Department of Pharmacology and Department of Medicine, MGM Medical College, Kamothe, Navi Mumbai. Two hundred twenty patients of Type II diabetes mellitus who conformed to the specified Inclusion and Exclusion Criteria were enrolled for the study after taking consent. A Case record form was administered to these patients, which included details regarding the prescription pattern, socio-demographic profile of the Type II diabetic patients.

Results: Average number of drugs per prescription was found to be 2.03 . None of the drugs were prescribed by generic name. Majority $(70 \%)$ of the patients were on combination therapy and $30 \%$ of patients were on antidiabetic monotherapy. Biguanides accounted for (41\%) of all the prescribed drugs followed by sulfonylureas (20\%), DPP4 inhibitors (19\%) and OHA combination therapy. Biguanides (Metformin) was the most commonly prescribed drug (84\%), followed by sulfonylureas (Glimepiride) (41\%) and DPP4 inhibitors [Teneligliptin (20\%), Vildagliptin (18\%)]

Conclusion: Metformin was the most frequently prescribed drug in diabetes. Prescription by Brand name is a matter of concern.

\section{Abbreviations}

ADA: American Diabetes Association; DA: Diabetes Atlas; DM: Diabetes Mellitus, DU/DUE: Drug Utilization Evaluation; FDC: Fixed Dcombination; HbA1c: Glycated Hemoglobin; ICMR: Indian Council of Medical Research; IDF: International Diabetes Federation; INRUD: International Network of Rational Use of Drugs; MODY: Maturity Onset Diabetes of the Young; OHA/OHD: Oral Hypoglycemic Agent/Drugs; OPD: Outpatient Department; WHO: World Health Organization

\section{Introduction}

Diabetes has emerged as a major healthcare problem in India. According to Diabetes Atlas (DA) published by the International Diabetes Federation (IDF), there is an alarming rise in disease progression from 40 million in 2007 to 70 million by 2025 in India and every fifth person with diabetes will be an Indian. A projected to rise from 171 million in 2000 to 366 million in 2030 is noted worldwide. The urban population in developing countries is projected to double between 2000 and 2030 [1]. The World Health Organization predicted a 50\% increase in deaths from diabetes over next 10 years, and by 2030, diabetes is projected to be the seventh leading cause of death. These estimated extrapolations and predictions are worrisome statistics in relation to the potential burden that diabetes may impose upon the country [2].

Poor Glycemic control in diabetes mellitus can sometimes be prevented by rational use of oral Hypoglycemic Agents (OHAs) and insulin [3]. Rational use of the drugs is a defined as follows: "That patients receive medications appropriate to their clinical needs, in doses that meet their own individual requirements for an adequate period of time and at the lowest cost to them and their community" [4]. Rational use of the drugs in populations can be effectively evaluated with drug utilization studies. The World Health Organization (WHO) defines "drug utilization" as the marketing, distribution, prescription and use of the drugs in a society considering its medical, social, and economic 
consequences [5]. Drug utilization study of anti-diabetic agents is therefore of paramount importance to promote rational drug use in diabetics and make available valuable information for the healthcare team. This study assessed the pattern of drug usage among type II diabetic patients and analyzed the prescriptions according to WHO core drug prescribing indicators.

The aim of the study was to determine the drug utilization pattern in patients of Type II diabetes mellitusaccording to WHO Core prescribing indicators. Recently since 2009 a new class of antidiabetic drugs; the dipeptidyl peptidase inhibitors (DPP-IV Inhibitors) have been introduced in the market. This has broadened the choice for the treatment of diabetes. The study will help to identify any change if any in the prescription trends of antidiabetic drugs as monotherapy as well as combination therapy in light of the new drugs being introduced and widely being used clinically [4].

\section{Material and Methods}

Necessary approval from the Institutional Ethics Committee was obtained (Approval No: 2015/SC/48) before initiating the study.

\section{Study design}

The study was an Observational, Cross-sectional, Descriptive type of study

\section{Study Site}

Department Medicine, MGM Medical College, Kamothe, Navi Mumbai

\section{Sample size}

220 patients of Type II diabetes mellitus attending the Medicine OPD for consultation

\section{Study population}

Type II diabetes mellitus patients who conformed to the specified Inclusion and Exclusion Criteria were enrolled for the study.

\section{Inclusion criteria}

- Aged $\geq 18$ years of age who were diagnosed with Type II Diabetes Mellitus Prior treated with OHA alone/Insulin alone/ OHA + Insulin (monotherapy or combination therapy) for at least 4 months.

- Patients who agreed to sign the informed consent form

\section{Exclusion criteria}

- Patients with type 1 diabetes

- Pregnant women, including those with gestational diabetes

- Patients with diabetes secondary to other factors (e.g. malnutrition, infection, surgery)
- Patients who could not complete the questionnaire

- Hospitalized and/or with psychiatric disorder during the data collection time (since there is a diabetes self-care practices assessment)

\section{Case record form (CRF)}

A CRF was prepared to record the following information of the Type II diabetes patients attending the medicine outdoor department.

Prescription pattern according to WHO Core prescribing indicators

- $\quad$ Average drugs prescribed

- Generic name wise drug prescribed

- Antibiotics used

- Injections used

- Drugs listed in (Essential Drug List)-India (2011)

Socio-Demographic Profile

- Age and Gender of the Type II diabetic patients were noted

- Occupation, Education and Family Income was taken into consideration to classify the individuals as belonging to Upper, Upper Middle, Lower Middle, Upper Lower and Lower Socio-economic class according to Kuppuswamy's socio-economic status scale (Modified 2012)

\section{Treatment Modality}

- Oral hypoglycemic agents (OHA) alone (Monotherapy, Dual Therapy or Combination therapy). The utilization of the oral hypoglycemic agents (Biguanides, Sulfonylureas, Alpha glucosidase inhibitor, DPP-IV Inhibitor, Thiozolidinediones and Meglitinides was noted on the $\mathrm{CRF}$

- Insulin (Short acting, long acting and Pre-Mixed) alone

- OHA and Insulin combination therapy

\section{Study procedure}

All the above mentioned data and completed prescriptions were collected on predesigned case record form. The diagnosed patients of type II diabetes mellitus attending the medicine outdoor department was enrolled after explaining the aim of the study. Written informed consent was obtained from each patient. Prior approval of Institutional Ethics Committee, Hospital superintendent and from the head of the medicine department was obtained.

Patients receiving any of the anti-diabetic drugs or Insulin or both were included in the study irrespective of their gender. Anatomical therapeutic classification was used to designate each 
drug prescribed. The Anatomical Therapeutic Chemical (ATC) Classification System is used for the classification of active ingredients of drugs according to the organ or system on which they act and their therapeutic, pharmacological and chemical properties. It is controlled by the World Health Organization Collaborating Centre for Drug Statistics Methodology (WHOCC), and was first published in 1976

The data was collected; analyzed and appropriate statistics was applied to obtain meaningful information.

\section{Primary Outcomes}

- The prescribing pattern of antidiabetic drugs used as monotherapy and combination therapy

- Average drugs prescribed, name wise drug prescribed , antibiotics used, injections used and drugs prescribed that are listed in Essential Drug List-India (2011),

\section{Secondary Outcomes}

- The socio-demographic, Anthropometric, Profile of Type II diabetic patients.

\section{Data analysis and statistical methods}

Descriptive statistics was used to summarize patient's demographics and survey responses. Differences in HbA1c goal was evaluated by chi-square tests (categorical variables). When significant differences were found in chi-square tests, the Bonferroni function was used to assess individual differences. Independent $t$ test (measurement data) was used to assess the relationship between inadequate glycaemic control and potential influencing factors. $\mathrm{P}<0.05$ was considered as statistically significant. The software package SPSS (version 20.0) was used for all calculations.

\section{Results}

\section{WHO core prescribing indices}

Total numbers of anti-diabetic drugs prescribed were 447 . Average number of drugs encountered per prescription was found to be 2.03. None of the drugs were prescribed by generic name. Encounter with parenteral preparation (injection) was $12.27 \%$. Encounter with an antibiotic was nil. Drugs prescribed from national essential drug list were $61.74 \%$.

\section{Socio demographic profile of Type II diabetic patients}

- Age: This study included a total of 220 patients with Type 2 diabetic patients aged between 21 and 82 years, with a mean age (S.D.) of 53.7 (11.6) years.81(36.8\%) were below 50 years $68(30.9 \%$ ) were between age group 50-59 years and 71(32.3\%)were above 60 years. (Table 1)

- Gender: Out of 220 patients $126(57.3 \%)$ were male and $94(42.7 \%)$ female. The male to female ratio was found to be 1.34. (Table 1)

- Socio-economic class: Out of 220 patients, 33 (15\%) were from upper class, 102 (46.4\%) from upper middle class ,10 (4.5\%) from lower middle class 53 (24.1\%) from upper lower class and 22(10\%) belonged to the lower socio-economic class(Table 1)

\section{Treatment modality of Type II diabetic patients}

Out of the total diabetic patients, 203 (92.3\%) were on Oral Hypoglycemic Agents only and 17 (7.7\%) were on combined Oral Hypoglycemic Agents and Insulin therapy.

Drugs utilization study of antidiabetic Drugs

- Overall utilization of antidiabetic Drugs: As diabetes progresses, functional decline in beta cells is usually apparent, and the need for combination therapy is unavoidable. Therefore, combination modalities have become an integral part of diabetes management. The basic rationale for combination therapy is to provide additive effects with different mechanisms of action and to allow lower doses for disease management. Consistent with the same, in the present study, majority $(70 \%)$ of the patients was on combination therapy and $30 \%$ of patients were on monotherapy (Table 2, Figure 1).

As show in Figure 2 and Table 2, among the total hypoglycaemic agents, biguanides accounted for (41\%) of all the prescribed drugs, followed by sulfonylureas $(20 \%)$, Insulin was prescribed in $4 \%$ cases. Biguanides (Metformin) was the most commonly: 185 (85\%) prescribed drug, followed by sulfonylureas (Glimepiride) in 91 (41\%), DPP4 inhibitors (Teneligliptin, Vildagliptin)

\begin{tabular}{|c|c|c|c|c|}
\hline \multirow[t]{2}{*}{ Sr.No. } & \multicolumn{2}{|c|}{ Parameters } & \multicolumn{2}{|c|}{ Total } \\
\hline & & & N (Number) & $\%$ (Percentage) \\
\hline \multirow{3}{*}{1} & \multirow{3}{*}{ Age } & $<50$ Years & 81 & 36.8 \\
\hline & & 50-59 Years & 68 & 30.9 \\
\hline & & $\geq 60$ Years & 71 & 32.3 \\
\hline \multirow{2}{*}{2} & \multirow{2}{*}{ Gender } & MALE & 126 & 57.3 \\
\hline & & FEMALE & 94 & 42.7 \\
\hline \multirow{5}{*}{3} & \multirow{5}{*}{ Socio-economic Class } & UPPER & 33 & 15.0 \\
\hline & & UPPER MIDDLE & 102 & 46.4 \\
\hline & & LOWER MIDDLE & 10 & 4.5 \\
\hline & & UPPER LOWER & 53 & 24.1 \\
\hline & & LOWER & 22 & 10.0 \\
\hline
\end{tabular}

Table 2: Overall utilization of antidiabetic agents on the basis of total number of units prescribed.

\begin{tabular}{|c|c|c|c|c|}
\hline Antidiabetic Drug Class & Drug & ATC Code & $\begin{array}{c}\text { No of Units } \\
\text { prescribed }\end{array}$ & $\begin{array}{c}\text { Percentage } \\
\text { (N=220) }\end{array}$ \\
\hline Biguanides & Metformin & A10BA02 & 185 & 84 \\
\hline \multirow{2}{*}{ Sulfonylureas } & Glimepiride & A10BB01 & 91 & 41 \\
\cline { 2 - 5 } & Glipizide & A10BB07 & 00 & 00 \\
\hline & Glibenclamide & A10BB12 & 00 & 00 \\
\hline Alpha-glucosidase & Voglibose & A10BF03 & 23 & 10 \\
\hline inhibitor & Acarabose & A10BF01 & 00 & 00 \\
\hline \multirow{2}{*}{$\begin{array}{c}\text { DPP-4 inhibitor } \\
\text { Thiazolidinediones }\end{array}$} & Sitagliptin & A10BH01 & 00 & 00 \\
\hline & Teneligliptin & A10BH- & 45 & 20 \\
\hline Mildagliptin & A10BH02 & 40 & 18 \\
\hline Meglitinide & Repaglinide & A10BX02 & 31 & 7 \\
\hline Human-insulin & Human-insulin & A10AC01 & 17 & 8 \\
\hline & & & 15 & 003 \\
\hline
\end{tabular}


in $95(38 \%)$ among Type II diabetic patients encounters with insulin was17 (8\%).

- Monotherapy: Out of 65 patients on monotherapy, most commonly prescribed oral hypoglycemic agent was biguanides: 38 (58\%), followed by sulfonylureas 23 (35\%) Meglitinide 3 (5\%) and thiazolidinediones 1(2\%). There were no encounters with insulin.

- Combination therapy: Out of overall total encounters $(n=220)$, biguanides (Metformin) were the most commonly prescribed drug 147 (95\%), followed by sulfonylureas (Glimepiride), among combination therapy, the number of prescriptions with oral hypoglycemic agent and Insulin was 17 (11\%).

Among insulin therapy as shown in Figure 3, short acting insulin was most commonly prescribed. Insulin lispro is most commonly (76\%) prescribed insulin.

- Fixed drug combinations: Fixed drug combination prescribed were $132(60 \%)$ among total prescriptions. There were two type of fixed dose combinations prescribed: two drug combinations 109 (83\%) and three drug combination 23 (17\%).Among two drug combination: Metformin + Vildagliptinwas encountered in $40(37 \%)$, Metformin + Teneligliptinin $5(5 \%)$, Metformin + Glimepiride in $37(34 \%)$ and Metformin + Repaglinidein 27(25\%), cases with three drug combination Biguanide + sulfonylureas + Alfa glucosidase inhibitor was encountered in $23(100 \%)$ prescriptions. (Figure 4).

\section{Discussion}

Diabetes is a major healthcare problem in India. The WHO defines diabetes mellitus as "A chronic, metabolic disease characterized by elevated levels of blood glucose (or blood sugar), which leads over time to serious damage to the heart,

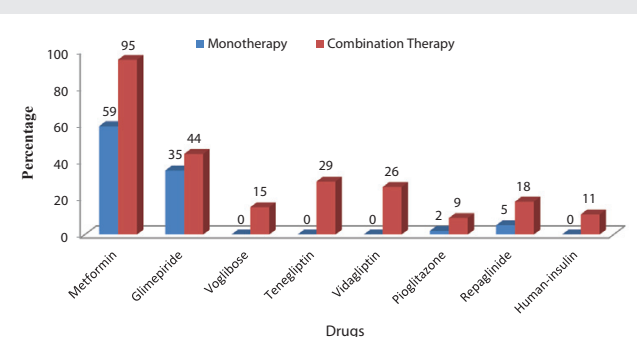

Figure 1: Pattern of antidiabetic drugs usage on the basis of type of therapy

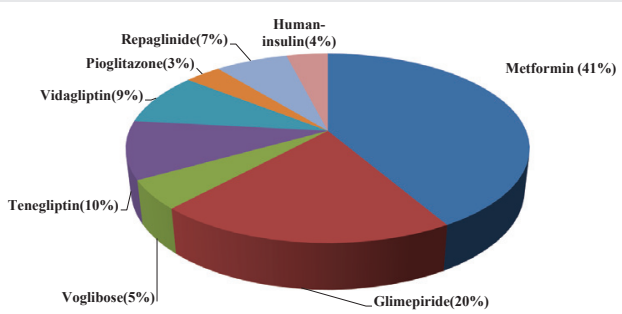

Figure 2: Overall utilization of antidiabetic agents

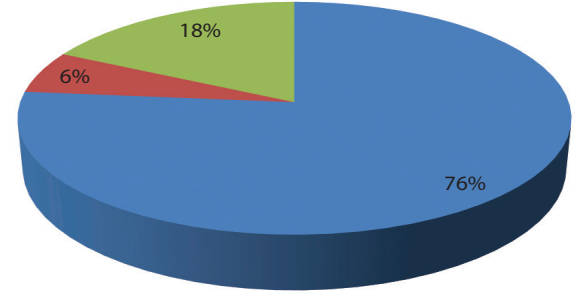

- Human Insulin lispro

- Human Insulin glargine

- Regular Human Insulin 50\% +NPH Human Insulin 50\%

Figure 3: Pattern of Insulin use.

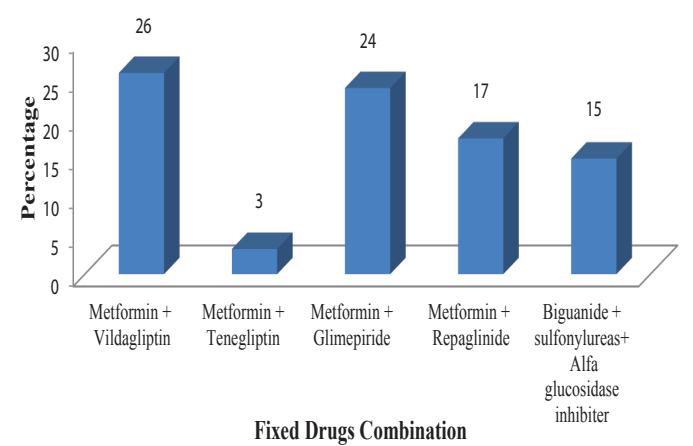

Figure 4: Pattern of anti-diabetic drug usage as fixed dose combination therapy.

blood vessels, eyes, kidneys, and nerves" [6]. A survey on Indian population shows that $4 \%$ of the adults suffered from diabetes mellitus in the year 2000 and it is expected to rise to $6 \%$ by the year 2025 [7]. In developing country like India, the majority of diabetics are in the age group of 45-64 years in contrast to developed countries where it is highly prevalent in age group more than 65 years of age [8]. According to Diabetes Atlas (ADA) published by the International Diabetes Federation (IDF), there is an alarming rise in disease progression from 40 million in 2007 to 70 million by 2025 in India and every fifth person in the word with diabetes will be an Indian [9]. Therefore studies focusing on various aspects of diabetes mellitus and its management are of paramount importance.

The present study was a hospital based cross sectional study conducted in the diabetes clinic of tertiary care hospital focusing on drug utilization pattern among Type II diabetic patients. The primary objective of the study was to assess the antidiabetic drug prescription pattern among diabetic patients attending the medicine outpatient department.

\section{Profile of patients with Type II diabetes mellitus}

Two hundred and twenty patients participated in the present study .Majority of the respondents (36.8\%) belonged to the age group of below 50 years followed by age group above 60 year (32.3\%).This finding is inconsistent with study conducted by Angel Dominic et al. (2016) [10] and Jambu Jain et al. [11].

In the present study the male to female ratio was found to be 1.34 male patients $(57.3 \%)$ and female patients $(42.7 \%)$ \}. This trend is similar to various studies conducted in India such as Soumya mary alex et al. [12], reporting50.3\%, Khushali G. 
Acharya et al. [13], (50.4\%), Sasisekhar T.V.D et al. [14], (56\%) and Angel Dominic et al. [10].

In the present study majority of the patients belonged to the upper middle socio-economic class which is similar to study conducted by Mamata Sharma et al. [15]. These results do not concur with the findings reported by Shah et al. [16], who found nearly $60 \%$ of patients were from low socio-economic status

Most of the diabetic patients $(92.3 \%)$ were on Oral Hypoglycemic Agents only and $7.7 \%$ were on Insulin and oral hypoglycemic agent (OHA) combination therapy. Only insulin was not prescribed to any patient attending Out Patient Department whereas study conducted by Gopinath B et al. [17], states that in $66.4 \%$ of the cases OHA only, insulin in (2.8\%) and combination therapy (OHA + Insulin) in (30.8\%) cases was prescribed. Similar studies have been reported by various authors in literature. Khushali G. Acharya et al. [18], Jambu Jain el al (2016) [11], Sushrut Varun Satpathy et al. [19], have also reported that OHA is the most commonly prescribed therapy

\section{Drug utilization study}

This study focused on the prescription pattern among diabetic patients attending the outpatient departments in the hospital the principal aim of drug utilization research is to facilitate the rational use of drugs in populations. For the individual patient, the rational use of a drug implies the prescription of a well-documented drug at an optimal dose, together with the correct information, at an affordable price. Knowledge of how drugs are being prescribed and used, will help to identify issues if any addressing rational drug use or suggest measures to improve prescribing habits [20]. With this point of view the study was designed.

Result demonstrated that the average number of drugs encountered per prescription was found to be 2.03. In this study, average number of drugs prescribed is less as compared to result of Upadhyay DK et al. [21], (3.76 per prescription) and V. Karthikeyan et al. [22], (4.83 per prescription). However the average number of drug prescribed is more compared to that reported by Das Priyaet al. [23], (1.83 per prescription), Kannan et al. [24], (1.4 per prescription) and Akshay A. Agarwalet al. [12], (1.4 per prescription). Total numbers of anti-diabetic drugs prescribed were 447 similar number of drugs was prescribed in study by Dev Priya et al. [23], (475 drugs). More number of drugs was prescribed in study by V. Karthikeyan et.al. [22], (1232 drugs) whereas less number of drugs was prescribed in study by Kumar Raj et al. [25], (366 drugs).

Drugs prescribed from national essential drug list were $61.74 \%$ which was more reported in study conducted by V. Karthikeyan et.al. [22] (74.30\%) but less (31.36\%).than published in the study conducted by Kumar Raj et.al. [25] In the present study no drug was prescribed by generic name. All were prescribed by brand names. Medical Council of India (MCI) called upon the doctors practicing medicine to prescribe Drugs with Generic names, as far as possible. The MCI circular reinforced that all Registered Medical Practitioners under the
Indian Medical Council (Professional Conduct, Etiquette and Ethics) Regulations, 2002 should comply with it without fail. Inspite of these regulations, prescription by brand name is a matter of concern.These results concur with finding observed specially by Kumar Raj et al. [25], and Dev Priya et.al. [.23]. In our study Encounter with parenteral preparation (injection) was $12.27 \%$ this was more (17.04\%) in study by V. Karthikeyan et al. [22].

In the present study, it was found that $30 \%$ of patients were on monotherapy with oral hypoglycemic agent compared to $70 \%$ on combination therapy. Similar results were reported by study conducted in Kerala by Soumya Mary Alexet, et al. [13], (58.4\% polytherapy).In a study conducted in Tamil Nadu by Sivasankari V et al. [4], monotherapy, and two drug combination therapies were prescribed in $21.7 \%$ and $78.3 \%$ patients, respectively.

The most commonly prescribed anti-diabetic drug class was biguanides (Metformin) both as monotherapy and/or in combinationtherapy, Metformin accounted for (85\%) of the total drugs prescribed, followed by sulfonylureas (Glimepiride), (41\%) and then DPP4 inhibitors (Teneligliptin,Vildagliptin) .Similar result regardingbiguanides and sulfonylureas has been documented in study conducted by Soumya mary alex et al. [13], Akshay A. Agarwal et al. [12], Jambu Jain et al. [11],

Unlike sulfonylureas, thiazolidinediones, and insulin, metformin is weight neutral, which makes it an attractive choice for obese patients. Furthermore, the management of Type II diabetes can be complicated by hypoglycemia, which can seriously limit the pursuit of glycemic control. By decreasing excess hepatic gluconeogenesis without raising insulin levels, metformin rarely leads to significant hypoglycemia when used as a monotherapy. As a result, metformin is widely considered an ideal first-line agent for the treatment of Type 2 diabetes. In addition, the cost of metformin is very low, thus making it affordable by the patients in economically weak countries like India [13]

Interestingly results did not concur with the usage pattern of DPP4 inhibitors. Soumyamaryalex et al. [13], Akshay A. Agarwal et al. [12], Jambu Jain et al. [11], Documented less utilization patterns compared to that reported in the present study. Results emphasize the increase in clinical usage of this relatively new class of antidiabetic drugs. The potential benefits of DPP-4 inhibitors include their complementary mechanism of action with other antidiabetic medications, a favorable adverse-effect profile, and a neutral effect on weight. With a low risk of hypoglycemia, DPP-4 Inhibitors are advantageous for patients who are close to their target HbA1c but who continually experience elevated glucose levels after meals [26].

The most commonly prescribed fixed dose combination among two drug combination was found to be Metformin + Vildagliptin (37\%) followed by Metformin + Glimepiride (34\%). Among three drug combination Biguanide + sulfonylureas + Alfa glucosidase inhibitor were most frequently prescribed fixed dose combination. Akshay A. Agarwal et al. [12], Sushrut 
Varun Satpathy et al. [19], showed similar results formetformin combination. Yerramilli A et al. [27], reported that most commonly prescribed combinations were Metformin and DPP4 inhibitor $(62 \%)$ as 2nd line agent, Metformin + Sulphonylureas + DPP4 inhibitor (44\%) as 3rd line agents. The present study DPP4 inhibitors were initiated in patients with higher body mass index and Glycated hemoglobin greater than $9 \%$. However soumyamaryalex et al. [13], reported metformin +glimipirideas the most common combination prescribed.

Results showed that three types of Insulin were encountered in the present study .Among Insulin preparations: lispro $(5.90 \%)$, insulin glargine $(0.45 \%)$, Regular Human Insulin and NPH Human Insulin combination (1.36\%) was prescribed. Result obtained by Amandeep Singh et al. [28], show more short acting insulin was prescribed (lispro $2.2 \%+$ aspart $15.38 \%$ ) where's mixed insulin was not prescribed at all.

To summarize, among the total hypoglycaemic agents prescribed, biguanides accounted for $(41 \%)$ of all the prescribed drugs, followed by sulfonylureas (20\%), DPP4 inhibitors (19\%), Meglitinide (7\%) glucosidase inhibitors (5\%) and thiazolidinediones (3\%) insulin ( $4 \%)$. Among Fixed drug combination, prescription of Metformin + Vildagliptin was the most common. We could not analyze the doses of prescribed antidiabetic drugs, or evaluate the appropriateness of therapy. Despite these limitations, the present study showed the prescription pattern in practice for a large number of patients with type 2 diabetes. In conclusion, the antidiabetic prescribing trend has moved away from monotherapy with Sulphonyluras and toward combination therapies to achieve better glycemic control with increased use of Biguanides and DPP- 4 inhibitors.

\section{Conclusion}

Metformin was the most frequently prescribed drug in diabetes followed by sulfonylureas (Glimepiride).Among antidiabetic drugs used as monotherapy, most commonly utilized drug class was biguanides followed by sulfonylureas. Metformin with glimpiride was the most frequently prescribed combination therapy.

Among Fixed drug combination, prescription of Metformin + Vildagliptin was the most common. Majority of drugs were prescribed from national essential drug list. Average number of drugs per prescription was found to be 2.03.None of the drugs were prescribed by generic name. All drugs were prescribed with brand name.

\section{References}

1. Sutharson L, Hariharan RS, Vamsadhara C (2003) Drug Utilization Study in Diabetology outpatient of a tertiary Hospital. Indian Journal Pharmacology 35: 237-240. Link: https://goo.gl/cVwjje

2. Gupta M, Singh R, Lehl S (2015) Diabetes in India: a long way to go.Int J Sci Rep 1: 1-2. Link: https://goo.gl/ecGYXa

3. Hermansen K, Mortensen LS, Hermansen ML (2008) Combining insulins with oral antidiabetic agents: Effect on hyperglycemic control, markers of cardiovascular risk and disease. Vasc Health Risk Manag 4: 561-574. Link: https://goo.gl/ZbYhct
4. Sivasankari V, Manivannan E, Priyadarsini SP (2013) Drug utilization pattern of anti-diabetic drugs in a rural area of Tamilnadu, South India - A prospective, observational study. Int J Pharm Biol Sci 4: 514-519. Link: https://goo.gl/LBZilY

5. Gama H (2008) Drug utilization studies. Arq Med 22: 69-74. Link: https://goo.gl/d1 vljf

6. (2005) Diagnostic Criteria [internet] ICMR Guidelines for Management of Type 2 Diabetes. Link: https://goo.gl/OIX3z1

7. Day C (2001) The rising tide of type 2 diabetes. The British Journal of Diabetes \& Vascular Disease. 1: 37-43. Link: https://goo.gl/FqbRxU

8. King H, Aubert RE, Herman WH (1998) Global burden of diabetes, 1995-2025 Prevalence, numerical estimates, and projections. Diabetes Care 21: 1414 1431. Link: https://goo.gl/yAuqJC

9. Mohan V, Sandeep S, Deepa R, Shah B, Varghese C, et al. (2007) Epidemiology of type 2 diabetes: Indian scenario. Indian journal of medical research 125 217-230. Link: https://goo.gl/8YPKaQ

10. Dominic A, Joseph J, Augustin RM, Begum R, Nanjwade BK, et al. (2016) Study Of Drug Use Evaluation On Oral Antihyperglycemic Agents In Type 2 Diabetes Melliitus And Their Potential Drug-Drug Interactions. 5: 1884-1896 Link: https://goo.gl/vd6EPc

11. Jain J, Sharma P, Jain J, Raja M (2016) Utilization pattern of oral hypoglycemic agents for diabetes mellitus type 2 patients attending out-patient department at tertiary care centre in Bhopal. International Journal of Basic \& Clinica Pharmacology 5: 1826-1830. Link: https://goo.gl/o8iwec

12. Agarwal $A A$, Jadhav $P R$, Deshmukh $Y A$ (2014) Prescribing pattern and efficacy of anti-diabetic drugs in maintaining optimal glycemic levels in diabetic patients. Journal of basic and clinical pharmacy 5: 79-83. Link: https://goo.gl/MUZADB

13. Alex SM, Bs S, Smitha S, Kn J, Menon AS, et al. (2015) Drug Utilization Pattern Of Antidiabetic Drugs Among Diabetic Outpatients In A Tertiary Care Hospital. Asian Journal of Pharmaceutical and Clinical Research 8: 144-146. Link: https://goo.gl/wtDOQK

14. Sasisekhar TV, Shabana S, Bhargav SY (2013) Gender: Does it have role has role in glycaemic control and diabetic distress in type 2 diabetes. IOSR-JDMS 4: 48-51. Link: https://goo.gl/JD54Gd

15. Sharma M, Sharma K, Gaur K, Bedi R (2016) Socio demographic profile of Diabetic cases attended at Diabetic clinic of a tertiary hospital of western Rajasthan India. 2: 23-28 Link: https://goo.gl/MKze8M

16. Shah V, Kamdar P, Shah N (2009) Assessing the knowledge, attitudes and practice of type 2 diabetes among patients of Saurashtra region, Gujarat International journal of diabetes in developing countries 29: 118-122. Link: https://goo.gl/5F3vqs

17. Gopinath B, Sri Sai Prasad M, Jayarama N, Prabhakara K (2013) Study of factors associated with poor glycemic control in Type -2 Diabetic patients. Global journal of medicine and public health.

18. Acharya KG, Shah KN, Solanki ND, Rana DA (2013) Evaluation of antidiabetic prescriptions, cost and adherence to treatment guidelines: A prospective, cross-sectional study at a tertiary care teaching hospital. Journal of basic and clinical pharmacy 4: 82-87. Link: https://goo.gl/5nJunC

19. Satpathy SV, Dutta S, Upreti B, Bhutia N, Sharma DK, eta al. (2014) Utilization and Adverse reaction profile of Anti-diabetic drugs in a Tertiary Care Hospital in East Sikkim: A Cross-Sectional Observational Study. In Indian journal of pharmacology 8: 223-228. Link: https://goo.gl/xh7RnK

20. (2003) Introduction to drug utilization research. 1st ed. Geneva: World Health Organization Link: https://goo.gl/8Ze41H 
21. Upadhyay DK, Palaian S, Ravi Shankar P, Mishra P, Sah AK, eta al. (2007) Prescribing pattern in diabetic outpatients in a tertiary care teaching hospital in Nepal. Journal of clinical and diagnostic research 1: 248-255. Link: https://goo.gl/WmUQFn

22. Karthikeyan V, Maadhusudhan S, Selvamuthukumran S (2016) Studies on Prescribing Pattern in the Management of Diabetes Mellitus in Rural Teaching Hospital, Saudi J. Med. Pharm. Sci 2: 100-107. Link: https://goo.gl/C9nEAV

23. Priya D, Purohit S, Pandey BL, Mishra S (2015) Evaluation of Antidiabetic Prescriptions from Medical Reimbursement Applications at Banaras Hindu University Health Care Facility. Journal of Pharmaceutical Care 2: 49-54. Link: https://goo.gl/4bkwDl

24. Principal JK (2011) A study on drug utilization of oral hypoglycemic agents in Type-2 diabetic patients. Asian Journal of Pharmaceutical and Clinical Research 4: 60-64. Link: https://goo.gl/OxJ0pr

25. Bhardwaj RA. (2013) A Study Of Drug Prescribing Pattern And Cost Analysis
Among Diabetic Patients In A Tertiary Care Teaching Institute In North India'. Journal of drug delivery and therapeutics Link: https://goo.gl/F6bv6c

26. Rolee Pathak, Mary Barna Bridgeman (2010) Dipeptidyl Peptidase-4 (DPP-4) Inhibitors in the Management of Diabetes. Pharmacy and Therapeutics 35 509-513. Link: https://goo.gl/rJWOcz

27. Yerramilli A, Mushtaq S, Amreen S, Satyanarayana V, Ramakrishnan S, et al. (2014) A Study On The Current Prescribing Patterns Of Dipeptidyl Peptidase 4 Inhibitors In A Multi Speciality Hospital Outpatient Setting Asian Journal of Pharmaceutical and Clinical Research 7: 134-136. Link https://goo.gl/3bvX30

28. Singh A, Dutta SB, Varma A, Beg MA, Kumar H, et al. (2016) A drug utilization and pharmacoeconomic study of anti-diabetic drugs prescribed to type 2 diabetes mellitus patients visiting the medicine out-patient department of a tertiary care hospital of north India. International Journal of Basic \& Clinical Pharmacology 5: 1220-1227. Link: https://goo.gl/9qc8ZM

Copyright: (c) 2017 Ashutosh K, et al. This is an open-access article distributed under the terms of the Creative Commons Attribution License, which permits unrestricted use, distribution, and $\mathrm{r}$ eproduction in any medium, provided the original author and source are credited. 